\title{
Quantitative structure-activity relationship for prediction of the toxicity of polybrominated diphenyl ether (PBDE) congeners
}

\author{
Yawei Wang ${ }^{\mathrm{a}}$, Chunyan Zhao ${ }^{\mathrm{b}}$, Weiping Ma ${ }^{\mathrm{b}}$, Hanxia Liu ${ }^{\mathrm{a}}$, \\ Thanh Wang ${ }^{\text {a }}$, Guibin Jiang ${ }^{\mathrm{a}, *}$ \\ ${ }^{a}$ State Key Laboratory of Environmental Chemistry and Ecotoxicology, Research Center for Eco-Environmental Sciences, \\ Chinese Academy of Sciences, P.O. Box 2871, Beijing 100085, China \\ ${ }^{\mathrm{b}}$ Department of Chemistry, Lanzhou University, Lanzhou 730000, China
}

Received 28 June 2005; received in revised form 31 October 2005; accepted 5 November 2005

Available online 9 January 2006

\begin{abstract}
Levels of Polybrominated diphenyl ether (PBDEs) are increasing in the environment due to their use as flame retardants. The similarities of structure to polychlorinated biphenyl (PCB) congeners suggest that they may share similar toxicological properties, such as hepatic enzyme induction. In this work, quantitative structure-activity relationship (QSAR) models were constructed based on 406 descriptors for the logarithm of toxicology index (aryl hydrocarbon receptor relative binding affinities, AhR, $I$ ) of 18 PBDE congeners. The method used for building model is the Heuristic method, which is included in comprehensive descriptors for structural and statistical analysis (CODESSA) software. The best regression model involved four descriptors, which were related to the conformational changes, atomic reactivity, molecular electrostatic field, and non-uniformity of mass distribution in a molecule of PBDEs, etc. The high square of the correlation coefficient $R^{2}(0.903)$ showed the model was satisfactory.
\end{abstract}

(C) 2005 Elsevier Ltd. All rights reserved.

Keywords: Polybrominated diphenyl ethers; Quantitative structure-activity relationships; Prediction; Principal component analysis

\section{Introduction}

Polybrominated diphenyl ethers (PBDEs) are widespread used in abundance as flame-retardants in polymers found in textiles and electrical appliances (Renner, 2000). The commercial PBDEs mixtures are produced with three degrees of bromination: deca-, consisting of 97-98\% decabromodiphenyl ether (DeBDE); octa-, consisting of $10-12 \%$ hexabromodiphenyl ether (HxBDEs), $43-44 \%$ heptabromodiphenyl ether (HeptaBDE) and 31-35\% octabromodiphenyl (OcBDEs); and penta-, consisting of 50-62\% pentabromodiphenyl ether (PeBDEs) and 24-38\% tetrabromodiphenyl ethers (TeBDEs) (Darnerud et al., 2001). The

\footnotetext{
* Corresponding author. Tel.: +86106284 9334; fax: +861062849179. E-mail address: gbjiang@rcees.ac.cn (G. Jiang).
}

global demand for PBDEs has been estimated to be close to 70000 tones in 1999.

PBDEs have widely distributed in environmental matrix and other samples such as human milk, blood plasma, and adipose tissue (Norén and Meironyte, 2000; Booij et al., 2002; Hale et al., 2002; Song et al., 2004; Vives et al., 2004; Wolkers et al., 2004). Among the target PBDE congeners, BDE47 and $99\left(2,2^{\prime}, 4,4^{\prime}\right.$-tetraBDE and $2,2^{\prime}, 4,4^{\prime}, 5-$ pentaBDE) are the most frequently encountered ones in the environment (Lindstrom et al., 1999).

PBDEs are concerned due to their persistence, bioaccumulation, and possible adverse effects in wildlife and humans (Tjärnlund et al., 1998; Sjödin et al., 1999). The toxicology of PBDEs is currently under investigation. But evidences emerge that PBDEs may be developmental neurotoxicants, and may cause neurochemical and hormonal deficits (Eriksson et al., 2001; Viberg et al., 2002; Zhou et al., 2002; Branchi et al., 2003). EU has banned 
the use in all applications of penta-BDE and octa-BDE in the EU market. However, limited toxicological data are obtained for only the most prevalent individual PBDE congeners (e.g. BDE-47, BDE-99, and BDE-100) (Chen et al., 2001; Rahman et al., 2001). Thus, there is a need for prediction tools to study PBDEs' properties including retention behavior, properties, and activity/toxicity for which analytical standards are currently difficult to obtain, but yet for which environmental data and toxicology indices are needed. Other works have been published to discuss retention mechanisms of PBDEs on capillary column by quantitative structure-gas chromatography retention time models (MLR) technique (Ikonomou and Rayne, 2002; Rayne and Ikonomou, 2003a,b). Wong et al. determined vapor pressures of 23 PBDEs by a function of temperature with a gas chromatographic retention time (Wong et al., 2001).

Here, based on the toxicology index $(\mathrm{AhR}, \mathrm{p} I)$ of 18 PBDEs, we report a QSAR model for all 209 PBDE congeners by the Heuristic method of CODESSA (comprehensive descriptors for structural and statistical analysis) technique, to investigate the significant relationships between the quantitative structure parameters and toxicology index of PBDEs.

The software CODESSA, developed by Katritzky group (Katritzky et al., 1995; Katritzky et al., 1995-1997), has been applied successfully in a variety of QSAR analyses (Oblak et al., 2000; Katritzky and Tatham, 2001). It can calculate a comprehensive set of descriptors: constitutional descriptors, topological descriptors, geometrical descriptors, electrostatic descriptors, and semi-empirical quantum chemical descriptors (in this work, 406 descriptors were calculated).

\section{Materials and methods}

A toxicity data set of 18 PBDEs congeners was taken from the previous work done by Chen et al. (2001). The relative binding affinities (RBA) values $(I)$, which were expressed as $\mathrm{p} I(-\log I)$ (Table 1$)$ for $\mathrm{Ah}$ receptor binding of individual PBDEs congeners, were in the $\mu \mathrm{M}$ range, indicating weaker affinity than the reference toxicant TCDD. Values of these 18 PBDEs in Table 1 were binding affinities relative to TCDD; concentration of $\left[{ }^{3} \mathrm{H}\right]$-TCDD was $1.0 \mathrm{nM}$.

\subsection{Physicochemical property calculations}

Firstly, 19 descriptors of PBDEs were calculated by HyperChem Release 7.0 software (HyperChem, 2002). Molecular geometries were optimized by the Polak Ribiere algorithm (Press et al., 1986) until rms gradient $0.01 \mathrm{kcal} \AA$ $\mathrm{mol}^{-1}$. The physicochemical quantities were obtained from the thermodynamically most stable configurations of the 209 PBDEs by semi-empirical parameterization method (PM3). Nineteen indices included molecular energies, heat of formation, frontier orbital energies, total dipole moments, QSAR properties $\log P$, van der Waals volumes, and surface areas (Table 2).

\subsection{Principal component analysis (PCA)}

Principal component analysis (PCA) on the analytical data was executed to obtain a visual representation of the main characteristics. PCA is an unsupervised multivariate technique in which new variables (called principal components (PCs)) are calculated as linear combinations of the olds ones. When most of the variation is explained by the first two or three components, a two- or three-dimensional scattergram, plotting the values for the first two or three principal components of the data points can be obtained to give essential features of the multidimensional scatter (in this case, usually $\mathrm{PC} 1$ and $\mathrm{PC} 2$, which eigenvalues are $>1$, are considered to represent the main examined objects and variables). The combined plot of scores and loadings allow us to recognize groups of samples with similar behavior and the existing correlation among the original variables. In this paper, PCA were performed with SPSS 12.0 for Windows Release 12.0.0 (4 Sep. 2003, SPSS Inc., 1989-2003).

\subsection{Theory of CODESSA}

The structures of PBDEs were drawn with HyperChem Release 7.0 software and saved as the hin files for MOPAC (Stewart, 1989), then the hin files were transferred into software CODESSA to calculate all kinds of descriptors. CODESSA includes two advanced procedures for systematic development of multi-linear QSAR/QSPR equations: (i) the Heuristic method, and (ii) the best multi-linear regression method. The heuristic method for descriptor selection proceeds with a preselection of descriptors by sequentially eliminating descriptors which do not match any of the following criteria: (i) the $F$-test greater than one unit; (ii) $R^{2}$ value less than a value defined at the start (default 0.01); (iii) the student's $t$-test less than that defined (default 0.1); and (iv) duplicate descriptors having a higher squared inter-correlation coefficient than a predetermined level (usually 0.8 ). The next step involves correlation of the given property with (i) the top descriptor in the above list with each of the remaining descriptors, and (ii) the next one with each of the remaining descriptors, etc. The best pairs, as evidenced by the highest $F$-values in the twoparameter correlations, are chosen and used for further inclusion of descriptors in a similar manner. The goodness of the correlation is tested by the correlation coefficient $\left(R^{2}\right)$, the $F$-test $(F)$, and the squared standard error $\left(s^{2}\right)$. The stability of the correlations was tested against the cross-validated coefficient, $R_{\mathrm{cv}}^{2}$. The heuristic method's advantages are the high speed and no restrictions on the size of the data set. Besides, it will demonstrate which descriptors have bad or missing values, which descriptors are insignificant, and which descriptors are highly intercorrelated. So, in this paper, we selected the heuristic method to build the best QSAR models. 
Table 1

Experimental, predicted activities $(\mathrm{p} I)$, and residual values of PBDEs by CODESSA

\begin{tabular}{|c|c|c|c|c|}
\hline No. & $\mathrm{Br}$ sites & Pred. & Exp. & Diff. \\
\hline 1 & 2 & 3.767 & & \\
\hline 2 & 3 & 3.478 & & \\
\hline 3 & 4 & 3.766 & 3.886 & -0.120 \\
\hline 4 & $2,2^{\prime}$ & 3.641 & & \\
\hline 5 & 2,3 & 3.308 & & \\
\hline 6 & $2,3^{\prime}$ & 3.096 & & \\
\hline 7 & 2,4 & 3.888 & & \\
\hline 8 & $2,4^{\prime}$ & 3.327 & & \\
\hline 9 & 2,5 & 4.582 & & \\
\hline 10 & 2,6 & 3.561 & & \\
\hline 11 & $3,3^{\prime}$ & 2.830 & & \\
\hline 12 & 3,4 & 2.820 & & \\
\hline 13 & $3,4^{\prime}$ & 2.888 & & \\
\hline 14 & 3,5 & 4.142 & & \\
\hline 15 & $4,4^{\prime}$ & 3.429 & 3.420 & 0.009 \\
\hline 16 & $2,2^{\prime}, 3$ & 3.247 & & \\
\hline 17 & $2,2^{\prime}, 4$ & 3.577 & 3.638 & -0.061 \\
\hline 18 & $2,2^{\prime}, 5$ & 4.190 & & \\
\hline 19 & $2,2^{\prime}, 6$ & 4.025 & & \\
\hline 20 & $2,3,3^{\prime}$ & 2.856 & & \\
\hline 21 & $2,3,4$ & 3.449 & & \\
\hline 22 & $2,3,4^{\prime}$ & 2.875 & & \\
\hline 23 & $2,3,5$ & 4.361 & & \\
\hline 24 & $2,3,6$ & 3.307 & & \\
\hline 25 & $2,3^{\prime}, 4$ & 3.009 & & \\
\hline 26 & $2,3^{\prime}, 5$ & 3.313 & & \\
\hline 27 & $2,3^{\prime}, 6$ & 3.121 & & \\
\hline 28 & $2,4,4^{\prime}$ & 3.423 & 2.921 & 0.502 \\
\hline 29 & $2,4,5$ & 4.790 & & \\
\hline 30 & $2,4,6$ & 4.283 & & \\
\hline 31 & $2,4^{\prime}, 5$ & 3.432 & & \\
\hline 32 & $2,4^{\prime}, 6$ & 2.947 & & \\
\hline 33 & $2,3^{\prime}, 4^{\prime}$ & 2.553 & & \\
\hline 34 & $2,3^{\prime}, 5^{\prime}$ & 3.900 & & \\
\hline 35 & $3,3^{\prime}, 4$ & 2.300 & & \\
\hline 36 & $3,3^{\prime}, 5$ & 3.470 & & \\
\hline 37 & $3,4,4^{\prime}$ & 2.557 & & \\
\hline 38 & $3,4,5$ & 3.153 & & \\
\hline 39 & $3,4^{\prime}, 5$ & 3.977 & & \\
\hline 40 & $2,2^{\prime}, 3,3^{\prime}$ & 2.988 & & \\
\hline 41 & $2,2^{\prime}, 3,4$ & 3.140 & & \\
\hline 42 & $2,2^{\prime}, 3,4^{\prime}$ & 3.038 & & \\
\hline 43 & $2,2^{\prime}, 3,5$ & 4.110 & & \\
\hline 44 & $2,2^{\prime}, 3,5^{\prime}$ & 3.491 & & \\
\hline 45 & $2,2^{\prime}, 3,6$ & 3.766 & & \\
\hline 46 & $2,2^{\prime}, 3,6^{\prime}$ & 2.759 & & \\
\hline 47 & $2,2^{\prime}, 4,4^{\prime}$ & 3.541 & 3.252 & 0.289 \\
\hline 48 & $2,2^{\prime}, 4,5$ & 4.174 & & \\
\hline 49 & $2,2^{\prime}, 4,5^{\prime}$ & 3.794 & 4.174 & -0.380 \\
\hline 50 & $2,2^{\prime}, 4,6$ & 3.728 & & \\
\hline 51 & $2,2^{\prime}, 4,6^{\prime}$ & 3.934 & & \\
\hline 52 & $2,2^{\prime}, 5,5^{\prime}$ & 4.502 & & \\
\hline 53 & $2,2^{\prime}, 5,6^{\prime}$ & 4.645 & & \\
\hline 54 & $2,2^{\prime}, 6,6^{\prime}$ & 4.051 & & \\
\hline 55 & $2,3,3^{\prime}, 4$ & 1.778 & & \\
\hline 56 & $2,3,3^{\prime}, 4^{\prime}$ & 2.224 & & \\
\hline 57 & $2,3,3^{\prime}, 5$ & 3.454 & & \\
\hline 58 & $2,3,3^{\prime}, 5^{\prime}$ & 3.413 & & \\
\hline 59 & $2,3,3^{\prime}, 6$ & 3.384 & & \\
\hline 60 & $2,3,4,4^{\prime}$ & 1.976 & & \\
\hline 61 & $2,3,4,5$ & 4.074 & & \\
\hline 62 & $2,3,4,6$ & 3.534 & & \\
\hline 63 & $2,3,4^{\prime}, 5$ & 3.426 & & \\
\hline
\end{tabular}

Table 1 (continued)

\begin{tabular}{|c|c|c|c|c|}
\hline No. & $\mathrm{Br}$ sites & Pred. & Exp. & Diff. \\
\hline 64 & $2,3,4^{\prime}, 6$ & 2.506 & & \\
\hline 65 & $2,3,5,6$ & 4.606 & & \\
\hline 66 & $2,3^{\prime}, 4,4^{\prime}$ & 2.472 & 2.699 & -0.227 \\
\hline 67 & $2,3^{\prime}, 4,5$ & 3.519 & & \\
\hline 68 & $2,3^{\prime}, 4,5^{\prime}$ & 3.528 & & \\
\hline 69 & $2,3^{\prime}, 4,6$ & 3.197 & & \\
\hline 70 & $2,3^{\prime}, 4^{\prime}, 5$ & 2.660 & & \\
\hline 71 & $2,3^{\prime}, 4^{\prime}, 6$ & 3.843 & 3.867 & -0.024 \\
\hline 72 & $2,3^{\prime}, 5,5^{\prime}$ & 4.003 & & \\
\hline 73 & $2,3^{\prime}, 5^{\prime}, 6$ & 3.840 & & \\
\hline 74 & $2,4,4^{\prime}, 5$ & 4.048 & & \\
\hline 75 & $2,4,4^{\prime}, 6$ & 3.071 & 3.398 & -0.327 \\
\hline 76 & $2,3^{\prime}, 4^{\prime}, 5^{\prime}$ & 3.721 & & \\
\hline 77 & $3,3^{\prime}, 4,4^{\prime}$ & 2.365 & 2.658 & -0.293 \\
\hline 78 & $3,3^{\prime}, 4,5$ & 3.451 & & \\
\hline 79 & $3,3^{\prime}, 4,5^{\prime}$ & 2.621 & & \\
\hline 80 & $3,3^{\prime}, 5,5^{\prime}$ & 3.653 & & \\
\hline 81 & $3,4,4^{\prime}, 5$ & 3.153 & & \\
\hline 82 & $2,2^{\prime}, 3,3^{\prime}, 4$ & 1.725 & & \\
\hline 83 & $2,2^{\prime}, 3,3^{\prime}, 5$ & 3.495 & & \\
\hline 84 & $2,2^{\prime}, 3,3^{\prime}, 6$ & 2.447 & & \\
\hline 85 & $2,2^{\prime}, 3,4,4^{\prime}$ & 1.970 & 1.721 & 0.249 \\
\hline 86 & $2,2^{\prime}, 3,4,5$ & 3.394 & & \\
\hline 87 & $2,2^{\prime}, 3,4,5^{\prime}$ & 2.287 & & \\
\hline 88 & $2,2^{\prime}, 3,4,6$ & 2.864 & & \\
\hline 89 & $2,2^{\prime}, 3,4,6^{\prime}$ & 2.679 & & \\
\hline 90 & $2,2^{\prime}, 3,4^{\prime}, 5$ & 3.566 & & \\
\hline 91 & $2,2^{\prime}, 3,4^{\prime}, 6$ & 2.650 & & \\
\hline 92 & $2,2^{\prime}, 3,5,5^{\prime}$ & 4.229 & & \\
\hline 93 & $2,2^{\prime}, 3,5,6$ & 3.832 & & \\
\hline 94 & $2,2^{\prime}, 3,5,6^{\prime}$ & 4.155 & & \\
\hline 95 & $2,2^{\prime}, 3,5^{\prime}, 6$ & 3.355 & & \\
\hline 96 & $2,2^{\prime}, 3,6,6^{\prime}$ & 3.510 & & \\
\hline 97 & $2,2^{\prime}, 3,4^{\prime}, 5^{\prime}$ & 3.523 & & \\
\hline 98 & $2,2^{\prime}, 3,4^{\prime}, 6^{\prime}$ & 2.810 & & \\
\hline 99 & $2,2^{\prime}, 4,4^{\prime}, 5$ & 4.027 & 3.854 & 0.173 \\
\hline 100 & $2,2^{\prime}, 4,4^{\prime}, 6$ & 4.153 & 4.114 & 0.039 \\
\hline 101 & $2,2^{\prime}, 4,5,5^{\prime}$ & 4.283 & & \\
\hline 102 & $2,2^{\prime}, 4,5,6^{\prime}$ & 2.852 & & \\
\hline 103 & $2,2^{\prime}, 4,5^{\prime}, 6$ & 3.929 & & \\
\hline 104 & $2,2^{\prime}, 4,6,6^{\prime}$ & 4.028 & & \\
\hline 105 & $2,3,3^{\prime}, 4,4^{\prime}$ & 1.774 & & \\
\hline 106 & $2,3,3^{\prime}, 4,5$ & 2.988 & & \\
\hline 107 & $2,3,3^{\prime}, 4^{\prime}, 5$ & 2.591 & & \\
\hline 108 & $2,3,3^{\prime}, 4,5^{\prime}$ & 2.194 & & \\
\hline 109 & $2,3,3^{\prime}, 4,6$ & 3.074 & & \\
\hline 110 & $2,3,3^{\prime}, 4^{\prime}, 6$ & 2.308 & & \\
\hline 111 & $2,3,3^{\prime}, 5,5^{\prime}$ & 3.794 & & \\
\hline 112 & $2,3,3^{\prime}, 5,6$ & 3.257 & & \\
\hline 113 & $2,3,3^{\prime}, 5^{\prime}, 6$ & 3.317 & & \\
\hline 114 & $2,3,4,4^{\prime}, 5$ & 3.469 & & \\
\hline 115 & $2,3,4,4^{\prime}, 6$ & 2.650 & & \\
\hline 116 & $2,3,4,5,6$ & 4.653 & & \\
\hline 117 & $2,3,4^{\prime}, 5,6$ & 2.878 & & \\
\hline 118 & $2,3^{\prime}, 4,4^{\prime}, 5$ & 2.796 & & \\
\hline 119 & $2,3^{\prime}, 4,4^{\prime}, 6$ & 2.895 & 2.959 & -0.064 \\
\hline 120 & $2,3^{\prime}, 4,5,5^{\prime}$ & 3.957 & & \\
\hline 121 & $2,3^{\prime}, 4,5^{\prime}, 6$ & 3.954 & & \\
\hline 122 & $2,3,3^{\prime}, 4^{\prime}, 5^{\prime}$ & 2.341 & & \\
\hline 123 & $2,3^{\prime}, 4,4^{\prime}, 5^{\prime}$ & 2.600 & & \\
\hline 124 & $2,3^{\prime}, 4^{\prime}, 5,5^{\prime}$ & 3.685 & & \\
\hline 125 & $2,3^{\prime}, 4^{\prime}, 5^{\prime}, 6$ & 2.914 & & \\
\hline 126 & $3,3^{\prime}, 4,4^{\prime}, 5$ & 2.890 & 2.569 & 0.321 \\
\hline 127 & $3,3^{\prime}, 4,5,5^{\prime}$ & 3.712 & & \\
\hline
\end{tabular}


Table 1 (continued)

\begin{tabular}{|c|c|c|c|c|}
\hline$\overline{\text { No. }}$ & $\mathrm{Br}$ sites & Pred. & Exp. & Diff. \\
\hline 128 & $2,2^{\prime}, 3,3^{\prime}, 4,4^{\prime}$ & 2.789 & & \\
\hline 129 & $2,2^{\prime}, 3,3^{\prime}, 4,5$ & 2.866 & & \\
\hline 130 & $2,2^{\prime}, 3,3^{\prime}, 4,5^{\prime}$ & 2.161 & & \\
\hline 131 & $2,2^{\prime}, 3,3^{\prime}, 4,6$ & 2.949 & & \\
\hline 132 & $2,2^{\prime}, 3,3^{\prime}, 4,6^{\prime}$ & 2.838 & & \\
\hline 133 & $2,2^{\prime}, 3,3^{\prime}, 5,5^{\prime}$ & 3.858 & & \\
\hline 134 & $2,2^{\prime}, 3,3^{\prime}, 5,6$ & 2.996 & & \\
\hline 135 & $2,2^{\prime}, 3,3^{\prime}, 5,6^{\prime}$ & 3.298 & & \\
\hline 136 & $2,2^{\prime}, 3,3^{\prime}, 6,6^{\prime}$ & 3.366 & & \\
\hline 137 & $2,2^{\prime}, 3,4,4^{\prime}, 5$ & 3.134 & & \\
\hline 138 & $2,2^{\prime}, 3,4,4^{\prime}, 5^{\prime}$ & 2.362 & & \\
\hline 139 & $2,2^{\prime}, 3,4,4^{\prime}, 6$ & 2.993 & & \\
\hline 140 & $2,2^{\prime}, 3,4,4^{\prime}, 6^{\prime}$ & 2.480 & & \\
\hline 141 & $2,2^{\prime}, 3,4,5,5^{\prime}$ & 2.816 & & \\
\hline 142 & $2,2^{\prime}, 3,4,5,6$ & 3.954 & & \\
\hline 143 & $2,2^{\prime}, 3,4,5,6^{\prime}$ & 2.654 & & \\
\hline 144 & $2,2^{\prime} 3,4,5^{\prime}, 6$ & 3.649 & & \\
\hline 145 & $2,2^{\prime}, 3,4,6,6^{\prime}$ & 2.193 & & \\
\hline 146 & $2,2^{\prime}, 3,4^{\prime}, 5,5^{\prime}$ & 4.509 & & \\
\hline 147 & $2,2^{\prime}, 3,4^{\prime}, 5,6$ & 4.373 & & \\
\hline 148 & $2,2^{\prime}, 3,4^{\prime}, 5,6^{\prime}$ & 3.659 & & \\
\hline 149 & $2,2^{\prime}, 3,4^{\prime}, 5^{\prime}, 6$ & 3.262 & & \\
\hline 150 & $2,2^{\prime}, 3,4^{\prime}, 6,6^{\prime}$ & 3.787 & & \\
\hline 151 & $2,2^{\prime}, 3,5,5^{\prime}, 6$ & 3.990 & & \\
\hline 152 & $2,2^{\prime}, 3,5,6,6^{\prime}$ & 4.257 & & \\
\hline 153 & $2,2^{\prime}, 4,4^{\prime}, 5,5^{\prime}$ & 4.598 & 4.602 & -0.004 \\
\hline 154 & $2,2^{\prime}, 4,4^{\prime}, 5,6^{\prime}$ & 4.544 & 4.638 & -0.094 \\
\hline 155 & $2,2^{\prime}, 4,4^{\prime}, 6,6^{\prime}$ & 3.888 & & \\
\hline 156 & $2,3,3^{\prime}, 4,4^{\prime}, 5$ & 2.829 & & \\
\hline 157 & $2,3,3^{\prime}, 4,4^{\prime}, 5^{\prime}$ & 2.256 & & \\
\hline 158 & $2,3,3^{\prime}, 4,4^{\prime}, 6$ & 2.425 & & \\
\hline 159 & $2,3,3^{\prime}, 4,5,5^{\prime}$ & 3.069 & & \\
\hline 160 & $2,3,3^{\prime}, 4,5,6$ & 4.153 & & \\
\hline 161 & $2,3,3^{\prime}, 4,5^{\prime}, 6$ & 3.749 & & \\
\hline 162 & $2,3,3^{\prime}, 4^{\prime}, 5,5^{\prime}$ & 2.648 & & \\
\hline 163 & $2,3,3^{\prime}, 4^{\prime}, 5,6$ & 2.674 & & \\
\hline 164 & $2,3,3^{\prime}, 4^{\prime}, 5^{\prime}, 6$ & 2.450 & & \\
\hline 165 & $2,3,3^{\prime}, 5,5^{\prime}, 6$ & 3.736 & & \\
\hline 166 & $2,3,4,4^{\prime}, 5,6$ & 3.408 & & \\
\hline 167 & $2,3^{\prime}, 4,4^{\prime}, 5,5^{\prime}$ & 2.951 & & \\
\hline 168 & $2,3^{\prime}, 4,4^{\prime}, 5^{\prime}, 6$ & 2.678 & & \\
\hline 169 & $3,3^{\prime}, 4,4^{\prime}, 5,5^{\prime}$ & 3.463 & & \\
\hline 170 & $2,2^{\prime}, 3,3^{\prime}, 4,4^{\prime}, 5$ & 2.310 & & \\
\hline 171 & $2,2^{\prime}, 3,3^{\prime}, 4,4^{\prime}, 6$ & 2.388 & & \\
\hline 172 & $2,2^{\prime}, 3,3^{\prime}, 4,5,5^{\prime}$ & 3.162 & & \\
\hline 173 & $2,2^{\prime}, 3,3^{\prime}, 4,5,6$ & 2.927 & & \\
\hline 174 & $2,2^{\prime}, 3,3^{\prime}, 4,5,6^{\prime}$ & 3.431 & & \\
\hline 175 & $2,2^{\prime}, 3,3^{\prime}, 4,5^{\prime}, 6$ & 1.694 & & \\
\hline 176 & $2,2^{\prime}, 3,3^{\prime}, 4,6,6^{\prime}$ & 2.202 & & \\
\hline 177 & $2,2^{\prime}, 3,3^{\prime}, 4,5^{\prime}, 6^{\prime}$ & 2.255 & & \\
\hline 178 & $2,2^{\prime}, 3,3^{\prime}, 5,5^{\prime}, 6$ & 3.570 & & \\
\hline 179 & $2,2^{\prime}, 3,3^{\prime}, 5,6,6^{\prime}$ & 3.685 & & \\
\hline 180 & $2,2^{\prime}, 3,4,4^{\prime}, 5,5^{\prime}$ & 3.463 & & \\
\hline 181 & $2,2^{\prime}, 3,4,4^{\prime}, 5,6$ & 2.370 & & \\
\hline 182 & $2,2^{\prime}, 3,4,4^{\prime}, 5,6^{\prime}$ & 2.320 & & \\
\hline 183 & $2,2^{\prime}, 3,4,4^{\prime}, 5^{\prime}, 6$ & 3.613 & 3.602 & 0.011 \\
\hline 184 & $2,2^{\prime}, 3,4,4^{\prime}, 6,6^{\prime}$ & 3.089 & & \\
\hline 185 & $2,2^{\prime}, 3,3,5,5^{\prime}, 6$ & 2.630 & & \\
\hline 186 & $2,2^{\prime}, 3,4,5,6,6^{\prime}$ & 3.881 & & \\
\hline 187 & $2,2^{\prime}, 3,4^{\prime}, 5,5^{\prime}, 6$ & 4.576 & & \\
\hline 188 & $2,2^{\prime}, 3,4^{\prime}, 5,6,6^{\prime}$ & 4.017 & & \\
\hline 189 & $2,3,3^{\prime}, 4,4^{\prime}, 5,5^{\prime}$ & 4.525 & & \\
\hline 190 & $2,3,3^{\prime}, 4,4^{\prime}, 5,6$ & 3.094 & & \\
\hline 191 & $2,3,3^{\prime}, 4,4^{\prime}, 5^{\prime}, 6$ & 2.715 & & \\
\hline 192 & $2,3,3^{\prime}, 4,5,5^{\prime}, 6$ & 4.155 & & \\
\hline
\end{tabular}

Table 1 (continued)

\begin{tabular}{lllll}
\hline No. & Br sites & Pred. & Exp. & Diff. \\
\hline 193 & $2,3,3^{\prime}, 4^{\prime}, 5,5^{\prime}, 6$ & 4.293 & & \\
194 & $2,2^{\prime}, 3,3^{\prime}, 4,4^{\prime}, 5,5^{\prime}$ & 3.305 & \\
195 & $2,2^{\prime}, 3,3^{\prime}, 4,4^{\prime}, 5,6$ & 3.638 & \\
196 & $2,2^{\prime}, 3,3^{\prime}, 4,4^{\prime}, 5,6^{\prime}$ & 3.567 & \\
197 & $2,2^{\prime}, 3,3^{\prime}, 4,4^{\prime}, 6,6^{\prime}$ & 3.409 & \\
198 & $2,2^{\prime}, 3,3^{\prime}, 4,5,5^{\prime}, 6$ & 4.941 & \\
199 & $2,2^{\prime}, 3,3^{\prime}, 4,5,5^{\prime}, 6^{\prime}$ & 3.364 & \\
200 & $2,2^{\prime}, 3,3^{\prime}, 4,5,6,6^{\prime}$ & 2.175 & \\
201 & $2,2^{\prime}, 3,3^{\prime}, 4,5^{\prime}, 6,6^{\prime}$ & 2.337 & \\
202 & $2,2^{\prime}, 3,3^{\prime}, 5,5^{\prime}, 6,6^{\prime}$ & 3.868 & \\
203 & $2,2^{\prime}, 3,4,4^{\prime}, 5,5^{\prime}, 6$ & 4.965 & \\
204 & $2,2^{\prime}, 3,4,4^{\prime}, 5,6,6^{\prime}$ & 2.497 & \\
205 & $2,3,3^{\prime}, 4,4^{\prime}, 5,5^{\prime}, 6$ & 3.477 & \\
206 & $2,2^{\prime}, 3,3^{\prime}, 4,4^{\prime}, 5,5^{\prime}, 6$ & 3.787 & \\
207 & $2,2^{\prime}, 3,3^{\prime}, 4,4^{\prime}, 5,6,6^{\prime}$ & 2.390 & \\
208 & $2,2^{\prime}, 3,3^{\prime}, 4,5,5^{\prime}, 6,6^{\prime}$ & 2.613 & \\
209 & Deca- & 4.306 & \\
\hline
\end{tabular}

\section{Results and discussion}

\subsection{Results of PCA using physicochemical properties calculated by $P M 3$}

Result of PCA gives three significant PCs (eigenvalues $>1$ ), which explains $92.4 \%$ of the variation in the data $(75.3 \%, 11.6 \%$, and 5.5\%, respectively). Parameters influencing $\mathrm{PC} 1$ are surface area (Grid) (SAG), surface area (Approx.) (SAA), core-core interaction (CCIA), $\log P$, binding energy $(\mathrm{BE})$, polarizability $(P)$, refractivity $(R)$, van der waals volume $(V)$, heat of formation (HOF), $E_{\mathrm{LUMO}}, E_{\mathrm{HOMO}}$, hydration energy (HE), electronic energy (EE), etc. $\mathrm{PC} 2$ is positive by $Q_{\min }$ and $\Delta E$, negative by $Q_{\max }, E_{\mathrm{HOMO}}$, and HOF. PC3 is positively determined by dipole moment (DIP) and negatively by $Q_{\mathrm{min}}$. As shown in Fig. 1, nine clusters of PBDE congeners can be observed in the score plot of $\mathrm{PC} 1$ and $\mathrm{PC} 2$, corresponding to the different degrees of bromination, from mono- (left) to decaBDE (right). There are some crosses in tetra-BDE, pentaBDE, and hexa-BDE groups. For example, hexa-BDE166 falls together in penta-BDE group. This is similar to the results of the previous works for 154 PCBs (Andersson et al., 1996) and PBDEs (Harju et al., 2002). In PC2, the degree of ortho-bromination separates the congeners. Congeners having one ortho-bromination have higher scores, such as BDE27, 75, 116, 191, etc. The non-ortho and two ortho-bromination with lower are found in the opposite area, such as BDE54, 104, etc.

\subsection{Modeling quantitative structural-activity relationships by heuristic method of CODESSA}

By using the Heuristic method, regression models were developed for 18 PBDE congeners. The heuristic correlations performed for the whole set provided the optimal equations for different numbers of descriptors in the range of 1-6. To avoid the over-parameterization of the model, an increase of the $R^{2}$ value of less than 0.02 was chosen 
Table 2

Calculated physicochemical parameters with abbreviations

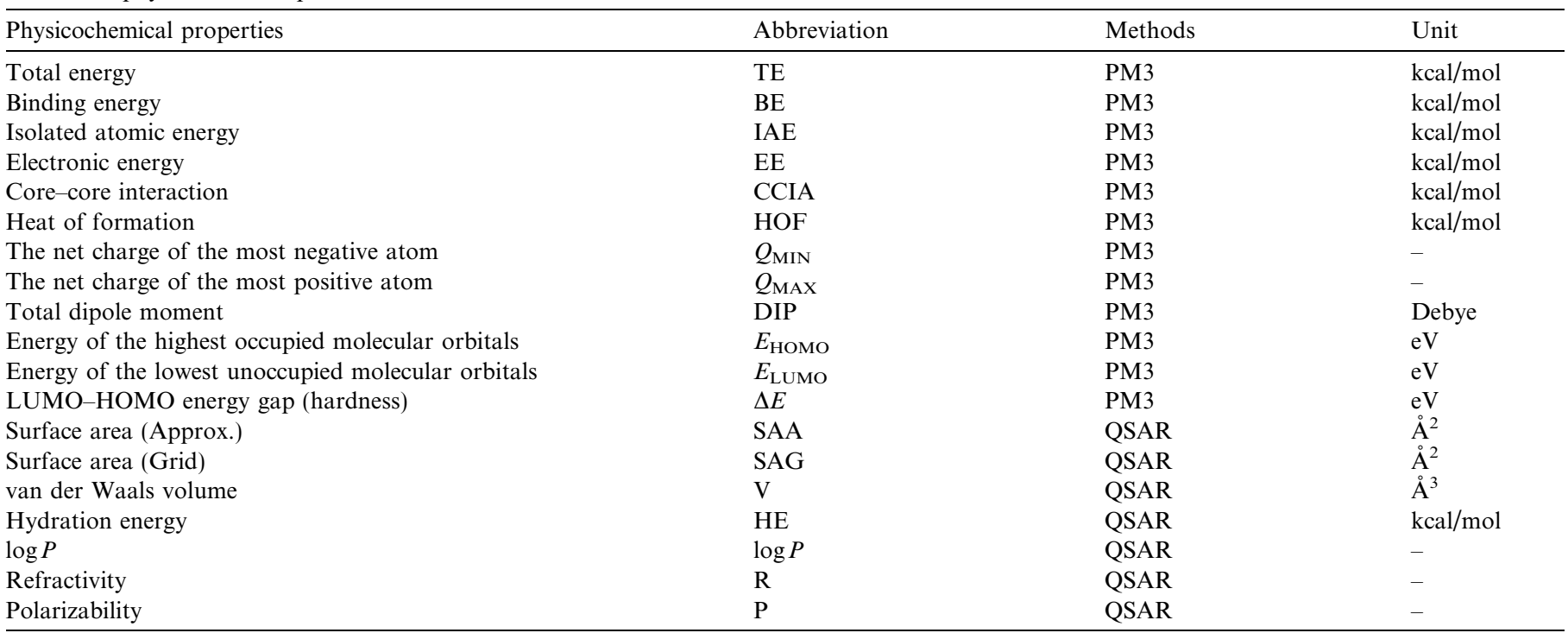
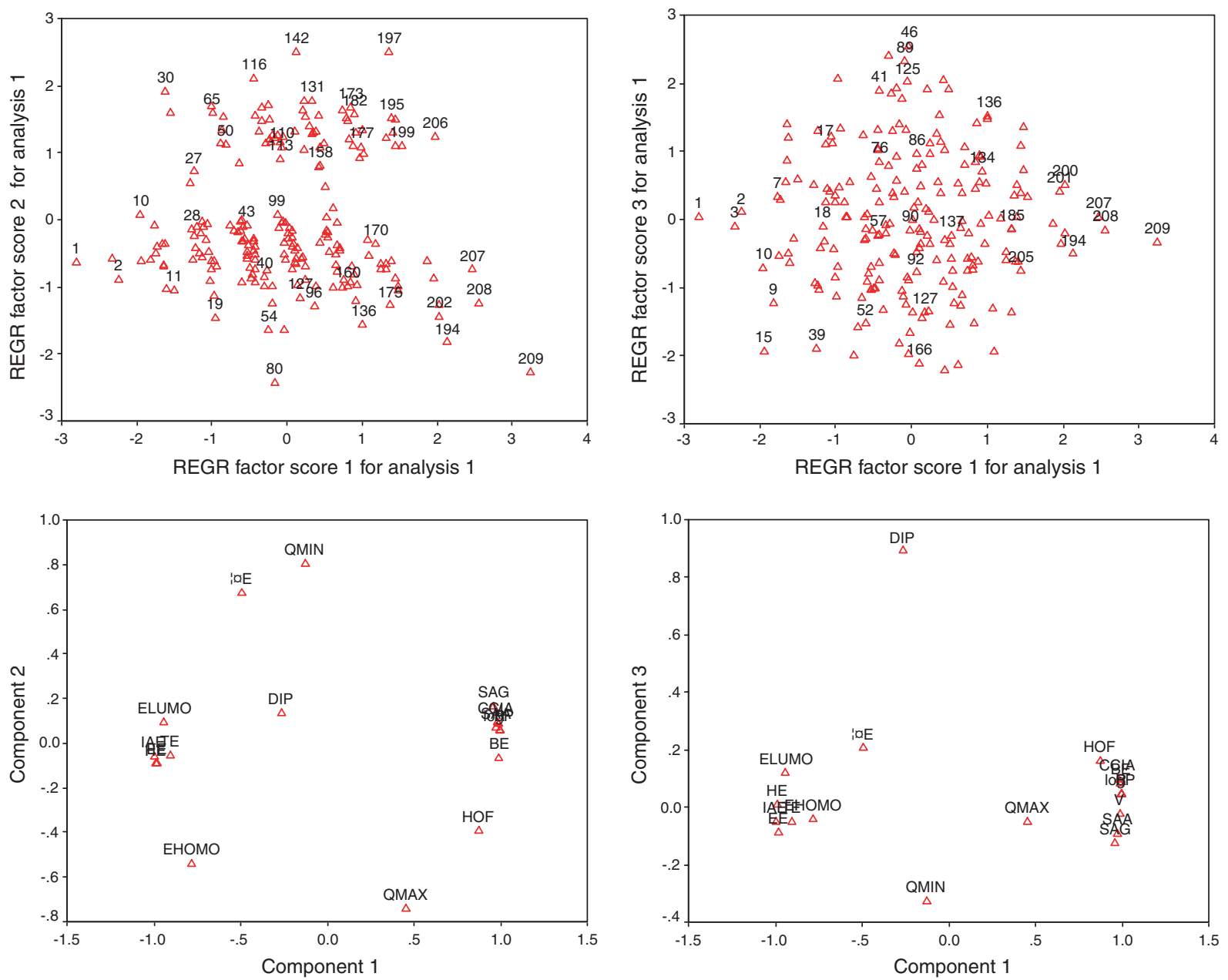

Fig. 1. PCA score plots of the first three principal components PCs (above), and corresponding loading plots (below) using the physicochemical properties calculated by PM3.

as the breakpoint criterion. From the viewpoint of statistics, the number of samples should be $4-5$ times of that of variables for linear regression. Plotting of $R^{2}$ and $R_{\mathrm{cv}}^{2}$ values against the number of descriptors (Fig. 2), which 


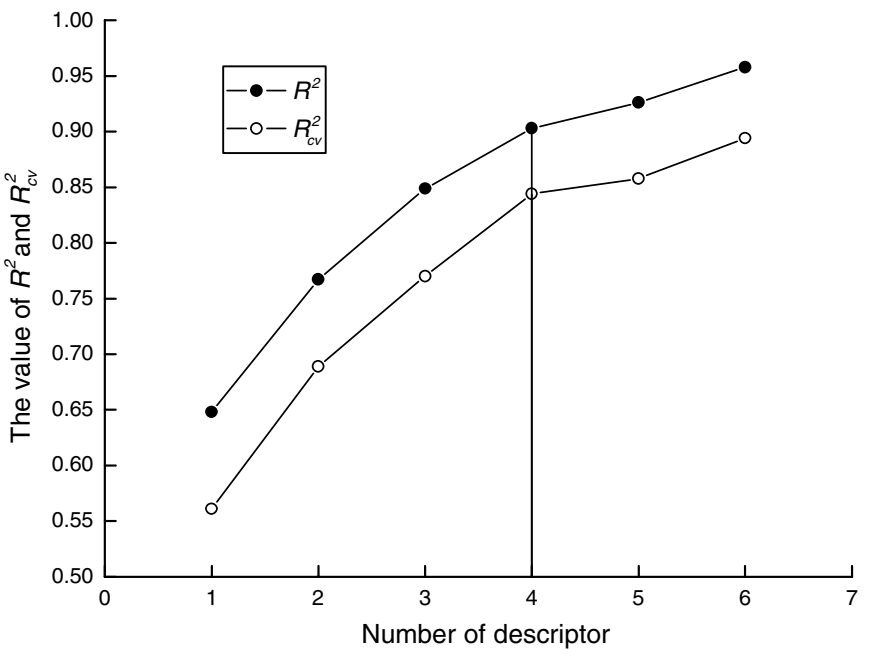

Fig. 2. Correlation coefficients $\left(R^{2}, R_{\mathrm{cv}}^{2}\right)$ versus number of descriptors. provide guidance regarding the number of descriptors to retain in the models, suggested that the best model contained four parameters. The experimental $\mathrm{p} I$ versus predicted $\mathrm{p} I$ of PBDEs and the residuals of the best regression model (Table 1) indicated that the best regression model (containing four descriptors) was very satisfactory. The descriptors involved in these correlations and the regression coefficients were listed in Table 3.

From Table 3 and Fig. 3 (relationship between $E_{\text {tot }}(\mathrm{C}-\mathrm{C})$ and $\mathrm{p} I$ ), we can see that Max total interaction for a $\mathrm{C}-\mathrm{C}$ bond $\left(E_{\mathrm{tot}}(\mathrm{C}-\mathrm{C})\right)$ (Strouf, 1986) (the first regression model, which contains one descriptor) is most important to the activity index $\mathrm{p} I$. This descriptor is defined as follows:

$E_{\text {tot }}(\mathrm{C}-\mathrm{C})=E_{\mathrm{c}}(\mathrm{C}-\mathrm{C})+E_{\text {exc }}(\mathrm{C}-\mathrm{C})$

where $E_{\mathrm{c}}(\mathrm{C}-\mathrm{C})$ is maximum coulomb interaction energy between two given atomic species $(\mathrm{C}$, here are carbon

Table 3

Regression models built by using heuristic method

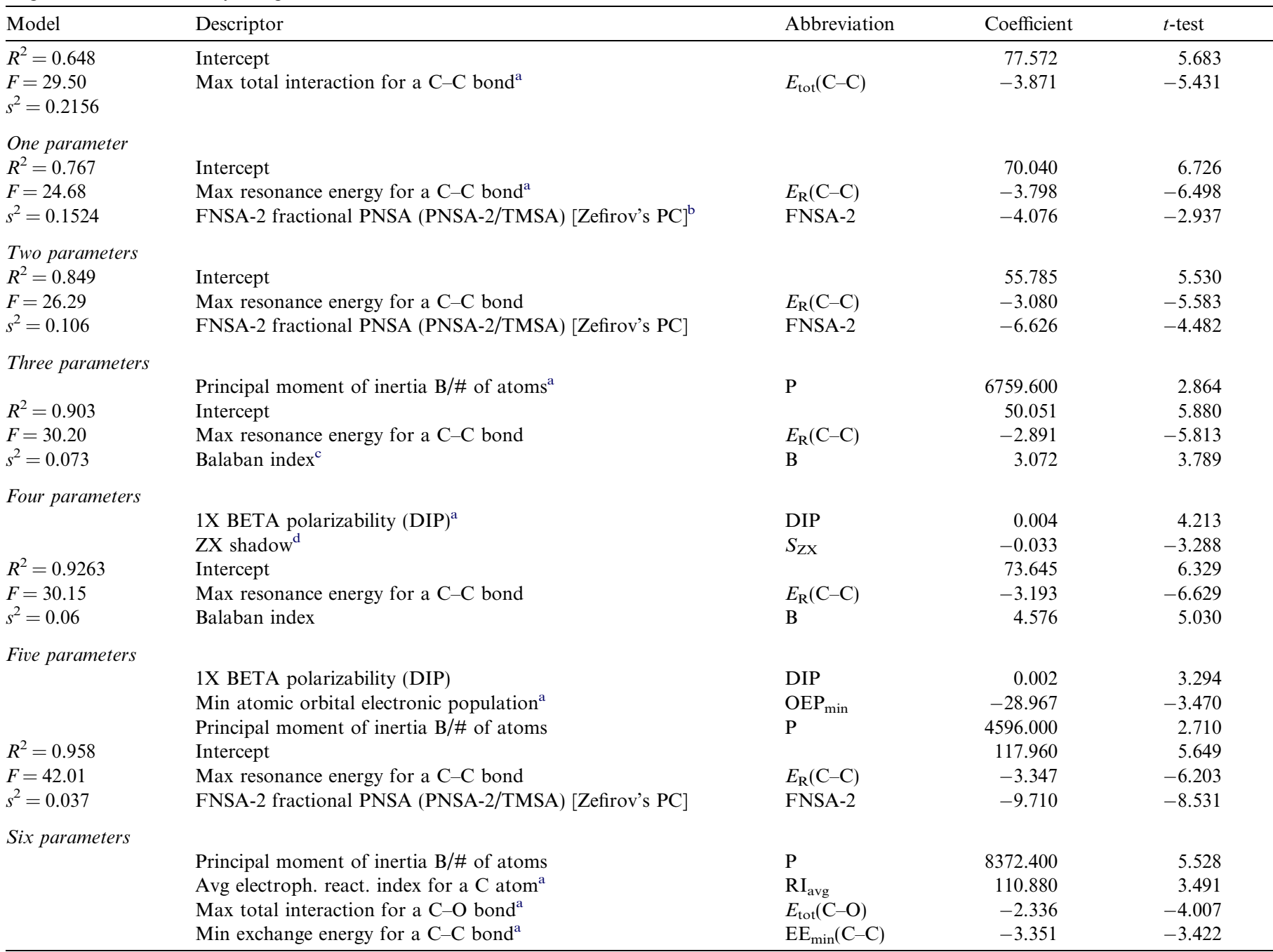

\footnotetext{
${ }^{a}$ Topological descriptor.

${ }^{\mathrm{b}}$ Geometrical descriptor.

${ }^{\mathrm{c}}$ Electrostatic descriptor.

${ }^{\mathrm{d}}$ Quantum-chemical descriptor.
} 


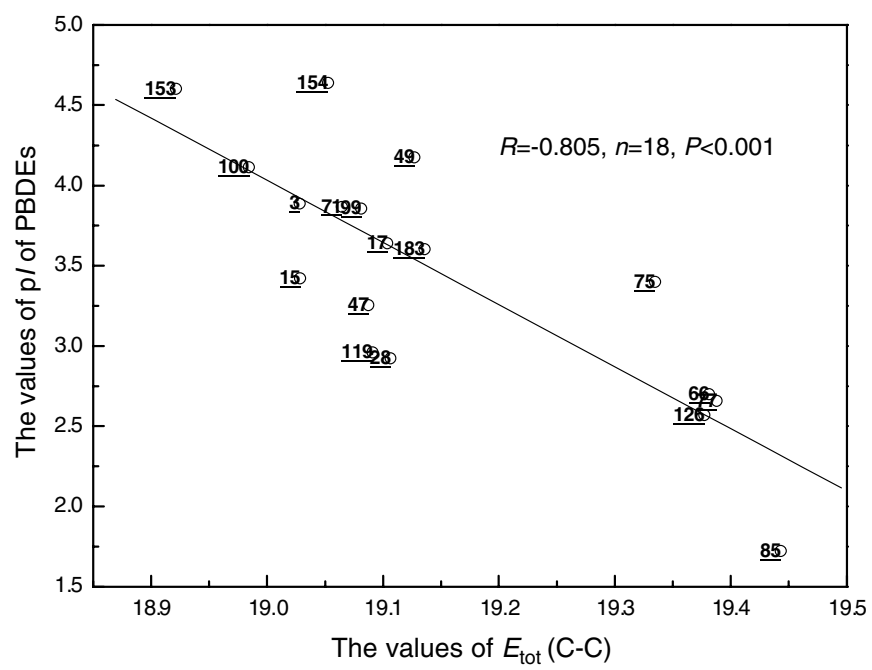

Fig. 3. Relationship between $E_{\text {tot }}(\mathrm{C}-\mathrm{C})$ and the values of $\mathrm{p} I$ of 18 PBDEs.

atoms) in the molecule, $E_{\text {exc }}(\mathrm{C}-\mathrm{C})$ is maximum electronic exchange energy between two given atomic species (here are carbon atoms) in the molecule. $E_{\mathrm{c}}(\mathrm{C}-\mathrm{C})$ is defined as

$E_{\mathrm{c}}(\mathrm{C}-\mathrm{C})=E_{\text {ee }}(\mathrm{C}-\mathrm{C})+E_{\mathrm{ne}}(\mathrm{C}-\mathrm{C})+E_{\mathrm{nn}}(\mathrm{C}-\mathrm{C})$

where $E_{\mathrm{ee}}(\mathrm{C}-\mathrm{C})$ is maximum electronic repulsion energy between two given atomic species (atom carbon) in the molecule; $E_{\text {ne }}(\mathrm{C}-\mathrm{C})$ is maximum nuclear-electron attraction energy between two given atomic species (atom carbon) in the molecule, and $E_{\mathrm{nn}}(\mathrm{C}-\mathrm{C})$ is maximum nuclear repulsion energy between two given atomic species (atom carbon) in the molecule, calculated as follows:

$$
\begin{aligned}
& E_{\mathrm{ee}}(\mathrm{C}-\mathrm{C})=\sum_{\mu v \in C} \sum_{\sigma \in C} P_{\mu v} P_{\lambda \sigma}\langle\mu v \mid \lambda \sigma\rangle \\
& E_{\mathrm{ne}}(\mathrm{C}-\mathrm{C})=\sum P_{\mu v}\left\langle\mu\left|Z_{\mathrm{C}} / R_{i \mathrm{C}}\right| v\right\rangle \\
& E_{\mathrm{nn}}(\mathrm{C}-\mathrm{C})=Z_{\mathrm{C}} Z_{\mathrm{C}} / R_{\mathrm{C}-\mathrm{C}} \\
& E_{\mathrm{exc}}(\mathrm{C}-\mathrm{C})=\sum_{\mu, v \in C} \sum_{\sigma \in C} P_{\mu v}\langle\mu v \mid \mu v\rangle
\end{aligned}
$$

where $P_{\mu v}$ and $P_{\lambda \sigma}$ are the density matrix elements and $\langle\mu v \mid \lambda \sigma\rangle$ are the electron repulsion integrals on the atomic basis; $Z_{\mathrm{C}}$ and $Z_{\mathrm{C}}$ are the nuclear (core) charges of atom carbon, respectively, and $R_{\mathrm{C}-\mathrm{C}}$ is the distance between them; $\left\langle\mu\left|Z_{\mathrm{C}} / R_{i \mathrm{C}}\right| v\right\rangle$ denote the nuclear-electron attraction integrals on the given atomic basis; $E_{\text {ee }}(\mathrm{C}-\mathrm{C})$, describes the electron repulsion driven processes in the molecule; $E_{\text {ne }}(\mathrm{C}-\mathrm{C})$, describes the nuclear-electron attraction driven processes in the molecule; $E_{\mathrm{nn}}(\mathrm{C}-\mathrm{C})$, describes the nuclear repulsion driven processes in the molecule. These three factors may be related to the conformational (rotational, inversional) changes or atomic reactivity in the molecule; $E_{\text {exc }}(\mathrm{C}-\mathrm{C})$, reflects the change in the Fermi correlation energy between the two electrons localized on atom carbon, respectively. It can be of importance in determining the conformational changes of the molecule and its spin properties.
So, $E_{\mathrm{tot}}(\mathrm{C}-\mathrm{C})$ is related to electron-electron, nuclearelectron, and nuclear-nuclear interaction in the molecule, and further related to the conformational (rotational, inversional) changes, atomic reactivity in the molecule, and molecular electrostatic field. The highest coefficient of $\mathrm{p} I$ and $E_{\text {tot }}(\mathrm{C}-\mathrm{C})$ suggest that conformation, atomic reactivity, and molecular electrostatic field should act an important role that influences the toxicity of PBDEs. The results of previous experimental and theoretical studies have also indicated that the interaction between the halogenated aromatic hydrocarbons (HAHs) and the AhR is a charge transfer type and that toxins appear to act as electron acceptors in the charge transfer complex (Poland et al., 1976; Miller et al., 1977; Cheney and Tolly, 1979; Bonati et al., 1994, 1995; Tuppurainen and Ruuskanen, 2000).

For 18 PBDE congeners, the best regression model can be described as follows:

$$
\begin{aligned}
\mathrm{p} I= & -2.891 E_{\mathrm{R}}(\mathrm{C}-\mathrm{C})+3.072 B+0.00406 \mathrm{DIP} \\
& -0.0334 D+50.051 \\
R^{2} & =0.903, R_{\mathrm{cv}}^{2}=0.844, n=18 \\
F & =30.20, s^{2}=0.073
\end{aligned}
$$

where $R$ is the correlation coefficient, $F$ is the value of the Fisher test, $s$ is the standard deviation of the fit, $R_{\mathrm{cv}}$ is the cross-validated correlation coefficient, and $n$ is the number of PBDE congeners; $E_{\mathrm{R}}(\mathrm{C}-\mathrm{C})$ is max resonance energy for a $\mathrm{C}-\mathrm{C}$ bond, which is highly correlated with $E_{\mathrm{tot}}(\mathrm{C}-\mathrm{C})$ (correlation coefficient $R$ is 0.911). They also represent the kinetic energy and electronuclear attraction energy associated with a charge distribution that lies between two atoms.

$B$ is Balaban index (Balaban, 1982). Balaban index is a measure of molecular "centricity", which describes the non-uniformity of mass distribution in a molecule. The value of the regression coefficient of Balaban index indicates the inferiority of cycle-symmetric structure to $\mathrm{p} I$, and superiority of cycle-symmetric structure to activities of PBDE congeners. DIP is $1 \mathrm{X}$ BETA polarizability (DIP), and $D$ is ZX shadow. ZX shadow (Rohrbaugh and Jurs, 1987), which codifies the size of the molecules, can be explained as the influence of steric factors on probability of forming a binding complex. The negative value of the respective correlation coefficient for ZX shadow indicates that the value $\mathrm{p} I$ declines as $\mathrm{ZX}$ shadow increases. So, an increase in this descriptor leads to an increase in the activities of PBDEs.

Using the best regression model, the Ah receptor relative binding affinities (RBA, $\mathrm{p} I$ ) of the remaining 191 PBDE congeners are predicted and list in Table 1.

\subsection{Results of PCA using molecular descriptors selected by regression models}

PCA was used for further study the relationship between descriptors and $\mathrm{p} I$ based on 11 descriptors included in six regression models. The first three significant PCs 


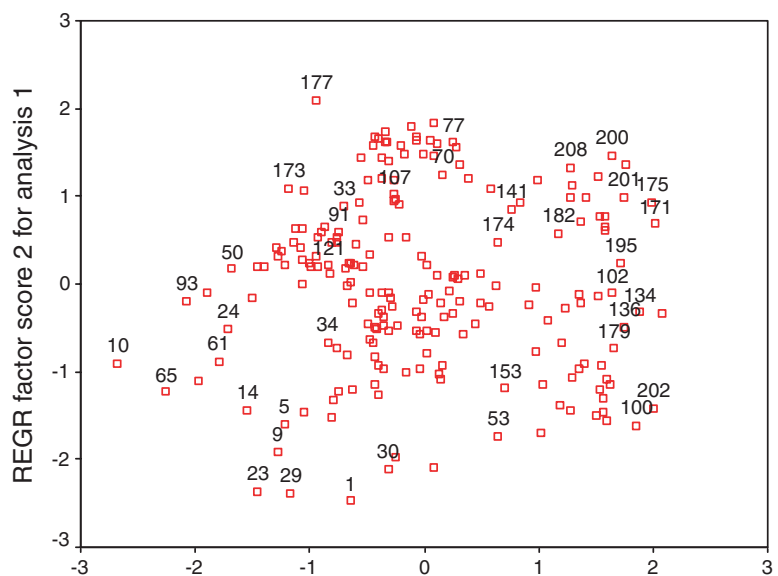

(a)

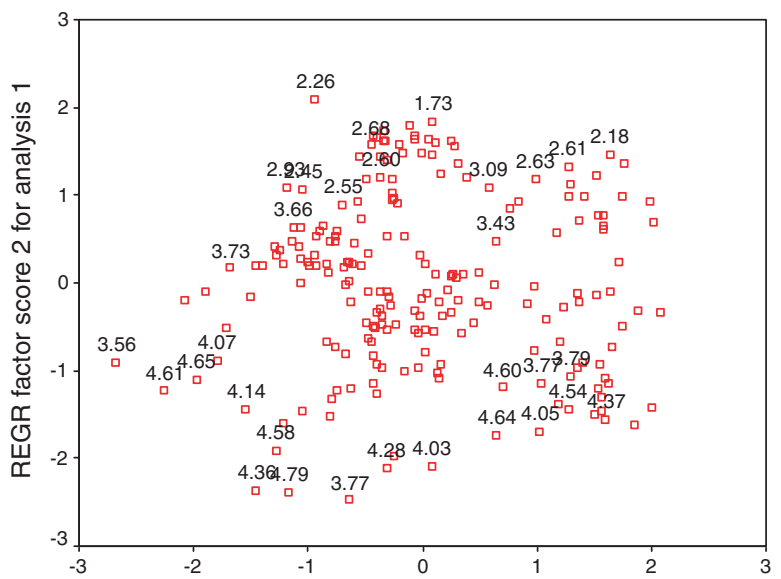

(b)

REGR factor score 1 for analysis 1

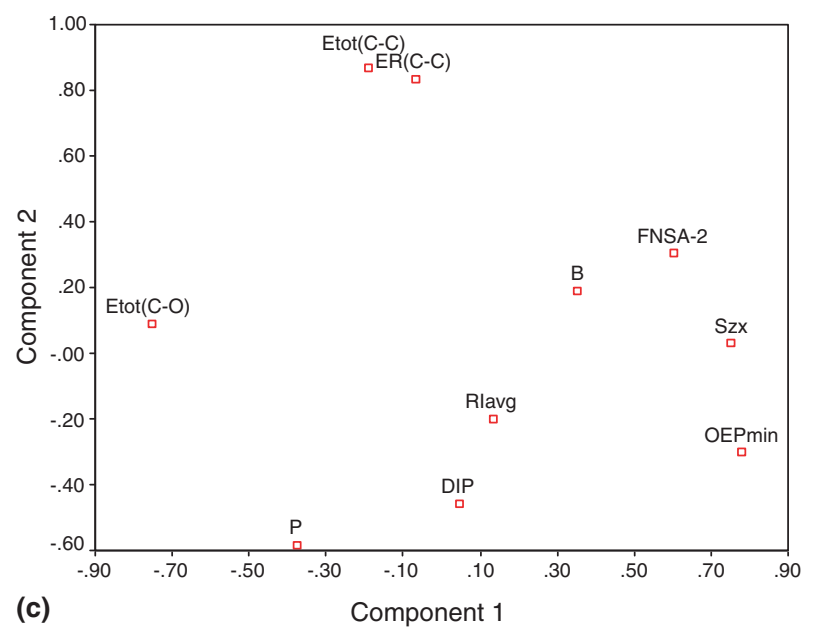

Fig. 4. PCA score plots and loading plot of the first two principal components PCs using molecular descriptors selected by regression models. (a) score plot labeled by IUPAC-based numbering (b) score plot labeled by the predicted $\mathrm{p} I$ of PBDEs congeners (c) loading plot of the first two principal components PCs using molecular descriptors selected by regression models.

(eigenvalues $>1$ ) of PCA explained $70.3 \%$ of the variation in the data $(31.1 \%, 30.0 \%$, and $9.2 \%$, respectively). The score and loading plots of $\mathrm{PC} 1$ vs. PC2 were shown in Fig. 4.

Comparing to the former results, the clusters of PBDE congeners in Fig. 4 are rather different from the clusters in Fig. 1. There are no obvious clusters of PBDEs corresponding to the different degrees of bromination. PBDEs with relative higher predictive values of $\mathrm{p} I$ distribute in the lower region of the score plot, and PBDEs with lower predictive values of $\mathrm{p} I$ exist in the upper region of the score plot. The loading plot suggests the higher predictive values of $\mathrm{p} I$ of PBDEs are negatively correlated with dipole moment, polarizability, and average electrophilic reactivity index for a $\mathrm{C}$ atom etc, but positively correlated with $E_{\mathrm{tot}}(\mathrm{C}-\mathrm{C})$ and $E_{\mathrm{R}}(\mathrm{C}-\mathrm{C})$.

Upper QSAR and PCA results show that the descriptors that represent the electronuclear attraction energy, polarizability, and the size of the molecules significantly affect the activity of PBDE congeners. This is consistent with the main theoretical models proposed for understanding the PCDD-AhR interaction: electrostatic and dispersion- type interaction models (Mhin et al., 2002). The electrostatic model is based on the effective interaction with the receptor and depends on the molecular electrostatic potential (MEP) around the ligand. The dispersion-type model considers the aromatic-aromatic interaction, which is related to the molecular polarizability and the distance between the receptor and the ligand.

\section{Conclusions}

Principal component analysis and QSAR models showed that retention behavior and properties such as vapor pressure of PBDEs were correlated with size/bulk of molecules, and activity/toxicity of PBDEs were influenced by conformational (rotational, inversional) changes, atomic reactivity in the molecule, and molecular electrostatic field. In contrast with PCBs, aryl hydrocarbon receptor relative binding affinities of PBDEs could not be related to the planarity of the molecule. This was possibly because the large size of the bromine atoms expanded the Ah receptor's binding site. In fact very little is still known about the 
toxicology of PBDEs, especially congener-specific data. It is possible that QSAR models could be expanded to obtain predicted toxicity data and to explain toxicology behavior of PBDEs.

\section{Acknowledgements}

This work was supported by the National Basic Research Program of China (2003CB415001). The authors would like to thank Prof. Man-cang Liu and his group (Department of Chemistry, Lanzhou University, China) for helpful discussions. The authors also thank Dr. Guosheng Chen (Environmental Health Centre, Ottawa, Canada) for supplying the toxicity data of PBDEs and the suggestion for this work.

\section{References}

Andersson, P., Haglund, P., Rappe, C., Tysklind, M., 1996. Ultraviolet absorption characteristics and calculated semiempirical parameters as chemical descriptors in multivariate modelling of polychlorinated biphenyls. J. Chemom. 10, 171-185.

Balaban, A.T., 1982. Highly discriminating distance-based topological index. Chem. Phys. Lett. 89, 399-404.

Booij, K., Zegers, B.N., Boon, J.P., 2002. Level of some polybrominated diphenyl ether (PBDEs) flame retardants along the Dutch coast as derived from their accumulation in SPMDs and blue mussels (Mytilus edulis). Chemosphere 46, 683-688.

Bonati, L., Fraschini, E., Lasagni, M., Pitea, D., 1994. Toward a mechanistic understanding of PCDD biological activity based on molecular electrostatic potential modeling. J. Mol. Struct. (THEOCHEM) 303, 43-54.

Bonati, L., Fraschini, E., Lasagni, M., Modoni, E.P., Pitea, D., 1995. A hypothesis on the mechanism of PCDD biological activity based on molecular electrostatic potential modeling. Part 2. J. Mol. Struct. (THEOCHEM) 340, 83-95.

Branchi, I., Capone, F., Alleva, E., Costa, L.G., 2003. Polybrominated diphenyl ethers: neurobehavioral effects following developmental exposure. Neurotoxicology 24, 449-462.

Chen, G.S., Chittim, B.G., Joyce, E.M., 2001. Synthesis of polybrominated diphenyl ethers and their capacity to induce CYP1A by the Ah receptor mediated pathway. Environ. Sci. Technol. 35, 37493756.

Cheney, B.V., Tolly, T., 1979. Electronic factors affecting receptor binding of dibenzo-p-dioxins and dibenzofurans. Int. J. Quantum Chem. 16, $87-110$.

Darnerud, P.O., Eriksen, G.S., Johannesson, T., Larsen, P.B., Viluksela, M., 2001. Environ. Health Perspect 109 (Suppl. 1), 49-68.

Eriksson, P., Jakobsson, E., Fredriksson, A., 2001. Brominated flame retardants: a novel class of developmental neurotoxicants in our environment. Environ. Health Perspect 109, 903-908.

Hale, R.C., La Guardia, M.J., Harvey, E.P., Mainor, T.M., Duff, W.H., Gaylor, M.O., 2002. Polybrominated diphenyl ether flame retardants in Virginia freshwater fishes (USA). Environ. Sci. Technol. 35, 4585 4591.

Harju, M., Andersson, P.L., Haglund, P., Tysklind, M., 2002. Multivariate physicochemical characterization and quantitative structureproperty relationship modeling of polybrominated diphenyl ethers. Chemosphere 47, 375-384.

HyperChem, 2002. Release 7.0 for Windows, Hypercube, Inc.

Ikonomou, M.G., Rayne, S., 2002. Chromatographic and ionization properties of polybrominated diphenyl ethers using GC/high-resolution MS with metastable atom bombardment and electron impact ionization. Anal. Chem. 74, 5263-5272.
Katritzky, A.R., Lobanov, V., Karelson, M., 1995. QSPR: The correlation and quantitative prediction of chemical and physical properties from structure. Chem. Soc. Rev. 24, 279-287.

Katritzky, A.R., Lobanov, V.S., Karelson, M., 1995-1997. Comprehensive descriptors for structural and statistical analysis, Reference Manual, Version 2.13.

Katritzky, A.R., Tatham, D.B., 2001. Theoretical descriptors for the correlation of aquatic toxicity of environmental pollutants by quantitative structure-toxicity relationships. J. Chem. Inf. Comput. Sci. 41, 1162-1176.

Lindstrom, G., Wingfors, H., Dam, M., vanBavel, B., 1999. Identification of 19 polybrominated diphenyl ethers (PBDEs) in long-finned pilot whale (Globicephala melas) from the Atlantic. Arch. Environ. Contam. Toxicol. 36, 355-363.

Mhin, B.J., Lee, J.E., Choi, W., 2002. Understanding the congener-specific toxicity in polychlorinated dibenzo-p-dioxins: chlorination pattern and molecular quadrupole moment. J. Am. Chem. Soc. 124, 144-148.

Miller, G., Sontum, S., Crosby, D.G., 1977. Electron-acceptor properties of chlorinated dibenzo-p-dioxins. Bull. Environ. Contam. Toxicol. 18, 611-616.

Norén, K., Meironyte, D., 2000. Certain Organochlorine and organobromine contaminants in Swedish human milk in perspective of past 20 30 years. Chemosphere 40, 1111-1123.

Oblak, M., Randic, M., Solmajer, T., 2000. Quantitative structure-activity relationship of flavonoid analogues. 3. Inhibition of p56lck protein tyrosine kinase. J. Chem. Inf. Comput. Sci. 40, 994-1001.

Poland, A., Glover, E., Kende, A.S., 1976. Stereospecific, high affinity binding of 2,3,7,8-tetrachlorodibenzo-p-dioxin by hepatic cytosol. Evidence that the binding species is receptor for induction of aryl hydrocarbon hydroxylase. J. Biol. Chem. 251, 4936-4946.

Press, W.H., Flannery, B.P., Teukolsky, S.A., Fetterling, W.T., 1986. Numerical Recipes. Cambridge University Press, Cambridge.

Rahman, F., Langford, K.H., Scrimshaw, M.D., Lester, J.N., 2001. Polybrominated diphenyl ether (PBDE) flame retardants. Sci. Total Environ. 275, 1-17.

Rayne, S., Ikonomou, M.G., 2003a. Development of a multiple-class highresolution gas chromatographic relative Retention time model for halogenated environmental contaminants. Anal. Chem. 75, 1049-1057.

Rayne, S., Ikonomou, M.G., 2003b. Predicting gas chromatographic retention times for the 209 polybrominated diphenyl ether congeners. J. Chromatogr. A. 1016, 235-248.

Renner, R., 2000. What Fate for Brominated Fire Retardants? Environ. Sci. Technol. 34, 223A-226A.

Rohrbaugh, R.H., Jurs, P.C., 1987. Descriptions of molecular shape applied in studies of structure/activity and structure/property relationships. Anal. Chim. Acta. 199, 99-109.

Sjödin, A., Hagmar, L., Klasson-Wehler, E., Kronholm-Diab, K., Jakobsson, E., Bergman, A., 1999. Flame retardant exposure: polybrominated diphenyl ethers in blood from Swedish workers. Environ. Health Perspect. 107, 643-648.

Song, W.L., Ford, J.C., Li, A., Mills, W.J., Buckler, D.R., Rock, K.J., 2004. Polybrominated diphenyl ethers in the Sediments of the Great Lakes. 1. Lake Superior. Environ. Sci. Technol. 38, 3286-3293.

Stewart, J.P.P., 1989. MOPAC, v.6.0, Quantum Chemistry Program Exchange, QCPE, No. 455, Indiana University, Bloomington, IN.

Strouf, O., 1986. Chemical Pattern Recognition. Research Studies Press, Letchworth.

Tjärnlund, U., Ericson, G., Örn, U., deWit, C., Balk, L., 1998. Effects of two polybrominated diphenyl ethers on rainbow trout (Oncorhynchus mykiss) exposed via food. Marine Environ. Res. 46, 107-112.

Tuppurainen, K., Ruuskanen, J., 2000. Electronic eigenvalue (EEVA): a new QSAR/QSPR descriptor for electronic substituent effects based on molecular orbital energies. A QSAR approach to the Ah receptor binding affinity of polychlorinated biphenyls (PCBs), dibenzo- $p$ dioxins (PCDDs) and dibenzofurans (PCDFs). Chemosphere 41, 843-848.

Viberg, H., Fredriksson, A., Eriksson, P., 2002. Neonatal exposure to the brominated flame retardant 2,2',4,4',5-pentabromodiphenyl ether 
causes altered susceptibility in the cholinergic transmitter system in the adult mouse. Toxicol. Sci. 67, 104-107.

Vives, I., Grimalt, J.O., Lacorte, S., Guillamón, M., Barceló, D., 2004. Polybromodiphenyl ether flame retardants in fish from Lakes in European High Mountains and Greenland. Environ. Sci. Technol. 38, 2338-2344.

Wolkers, H., van Bavel, B., Derocher, A.E., Wiig, O., Kovacs, K.M., Lydersen, C., Lindström, G., 2004. Congener-specific accumulation and food chain transfer of polybrominated diphenyl ethers in two Arctic Food Chains. Environ. Sci. Technol. 38, 1667-1674.

Wong, A., Lei, Y.D., Alaee, M., Wania, F., 2001. Vapor pressures of the polybrominated diphenyl ethers. J. Chem. Eng. Data. 46, 239242.

Zhou, T., Taylor, M.M., DeVito, M.J., Crofton, K.M., 2002. Developmental exposure to brominated diphenyl ethers results in thyroid hormone disruption. Toxicol. Sci. 66, 105-116. 